\title{
Étude par éléments finis du frottement latéral des pieux dans de l'argile
}

\section{P. J. BRUGGER}

F. R. LOPES

M. S. S. ALMEIDA

Université fédérale de Rio de Janeiro Caixa Postal 68506 Rio de Janeiro $R J$ - 21945, Brazil.
L'étude numérique du comportement du sol au voisinage d'un pieu sous chargement axial a été faite en utilisant la technique des éléments finis. Deux modèles de

comportement typiques en Mécanique des Sols ont été utilisés, à savoir un modèle élastique non linéaire et un modèle élasto-plastique basé sur la théorie de l'état critique. On examine en particulier, à partir de ces modèles, le cas de chargements drainés et non dirainés. L'étude réalisée montre en particulier que le problème du frottement latéral le long du fût du pieu est de nature cinématique et que seul un modèle élasto-plastique peut rendre compte des contraintes et des déformations développées dans le sol au voisinage du fût du pieu. Une discussion est présentée sur la modification de l'état de contrainte provoquée par la mise en place initiale du pieu.

\section{Finite element investigation of shaft friction of piles in clay}

An investigation of the soil behavior around a pile under axial loading is presented, based on a finite element analysis. Two classical constitutive models, typically used in soil mechanics are evaluated in a comparative way : a non-linear elastic model and an elasto-plastic critical state model. Both drained and

undrained analysis are carried out. The investigation shows that the problem of shaft friction along a pile is a kinematic controlled problem, and that only an elasto-plastic model can correctly represent stresses and strains developed in the soil adjacent to the pile shaft. A discussion on the stress state generated by the pile installation is also presented. 


\section{Introduction}

L'évaluation du frottement latéral limite mobilisable le long d'un pieu installé dans une argile est un sujet controversé depuis longtemps, et a attiré l'attention de nombreux chercheurs. Traditionnellement, le frottement latéral a été mis en relation avec la résistance au cisaillement non drainée du sol à laquelle est appliqué un facteur $\alpha$ de façon à prendre en considération les effets d'installation (Tomlinson, 1957 ; McClelland, 1969 ; Wroth, 1972). Cette technique est appelée « Méthode Alpha ».

Chandler (1968) a suggéré, pour la première fois, que le frottement latéral mobilisable le long d'un pieu correspondait à un cisaillement pur le long du fût et a proposé que son calcul soit fait à partir des contraintes effectives initiales $\sigma_{\text {ho }}^{\prime}$ et $\sigma_{\text {vo' }}^{\prime}$ existant dans le sol au repos

$$
\tau_{f}=\sigma_{\text {ho }}^{\prime} \tan \phi^{\prime}=\sigma_{\text {vo }}^{\prime} K_{o} \tan \phi^{\prime}
$$

Cette idée a été appuyée par Burland (1973), qui a réécrit l'équation (1) sous la forme suivante:

$$
\tau_{\mathrm{f}}=\beta \sigma_{\text {vo }}^{\prime}
$$

L'utilisation de l'équation (2) correspond à la méthode dite " Méthode Bêta ».

L'expression (1) implique que :

- des surpressions interstitielles ne sont pas générées pendant le chargement du pieu;

- la rupture survient sur un plan vertical par un mécanisme de glissement simple.

En examinant l'état des contraintes dans le sol au voisinage du fût du pieu, Parry et Swain (1977a, 1977b) ont observé que les conditions de rupture sont d'abord réalisées sur un plan incliné en relation avec le plan vertical et que, pour que la rupture se produise sur le plan vertical, une rotation des contraintes principales est nécessaire. D'après cette hypothèse, des équations ont été suggérées pour la résistance des argiles normalement consolidées et surconsolidées. Lopes (1979), par des analyses utilisant la méthode des éléments finis et un modèle élastique non linéaire, a observé que le sol au voisinage du fût du pieu est soumis à un cisaillement pur. Ceci explique pourquoi on n'observe pas de génération de surpressions interstitielles pendant le chargement dans des argiles normalement consolidées ou légèrement surconsolidées.

Martins et Potts (1983) ont fait des analyses à l'aide du modèle Cam-Clay modifié et ont confirmé la rotation des contraintes principales pour les différents états de contraintes initiales et ont observé que la contrainte de cisaillement maximale est proche de la résistance non drainée du sol avant le chargement. Cette observation explique le succès de la a Méthode Alpha » pour la prévision du frottement latéral des pieux.

Randolph et al. (1979) ont présenté une étude numérique du pieu basée sur l'utilisation du modèle élastoplastique Cam-Clay modifié. Les effets d'installation sont modélisés par l'expansion non drainée d'une cavité cylindrique suivie d'une phase de consolidation radiale. Les auteurs concluent que les variations de contraintes normées par la résistance au cisaillement non drainée initiale du sol vierge sont indépendantes de la valeur du degré de surconsolidation OCR et, qu'après la consolidation, la résistance non drainée augmente d'environ $60 \%$.
Le présent travail repose sur les résultats d'une recherche menée sur le comportement du sol au voisinage du fût du pieu pendant son chargement axial. On a utilisé la méthode des éléments finis et deux modèles classiques de sol. Le travail avait deux objectifs :

- analyser la performance des deux modèles numériques pour l'étude d'un problème géotechnique particulier;

- examiner le développement des contraintes et de la rupture du sol au voisinage du fût du pieu au cours de chargement drainé et non drainé.

Une discussion sur les états de contrainte induits par le processus d'installation du pieu est aussi présentée.

\section{2}

\section{Description des modèles utilisés et de la technique de résolution numérique}

Deux logiciels de calcul basés sur la méthode des éléments finis : PROGEO et CRISP ont été utilisés. PROGEO est destiné à simuler divers problèmes géotechniques à partir d'un modèle de sol élastique non linéaire et utilise une résolution incrémentale. Ce logiciel présente les autres caractéristiques suivantes:

- possibilité de simuler des conditions non drainées en contraintes totales ou effectives (dans le cas des contraintes effectives, les surpressions interstitielles sont calculées en utilisant les paramètres $\alpha$ et $\beta$ de Henkel) :

- la solution incrémentale utilise un schéma de type de Runge-Kutta (à deux pas) :

- utilisation d'un élément isoparamétrique à 8 nceuds.

En accord avec le modèle élastique non linéaire utilisé, le module tangent initial $E_{j}$ est lié à la contrainte moyenne effective par l'équation:

$$
E_{i}=K \sigma_{a}\left(\frac{\sigma_{o c t}^{\prime}}{\sigma_{a}}\right)^{n}
$$

avec:

$E_{\text {f }} \quad$ : module tangent initial

$\mathrm{K}$ : constante

$\sigma_{\mathrm{a}} \quad$ : pression atmosphérique

$\sigma_{\text {oc }}^{\prime}$ : contrainte moyenne effective (octaédrique)

n : exposant

Le module tangent $E_{\text {j }}$ est fonction des contraintes de cisaillement et est relié au module initial par l'équation hyperbolique suivante:

$$
E_{t}=E_{\mathrm{j}}\left[1-R_{\mathrm{f}} \frac{\sigma_{\mathrm{t}}-\sigma_{3}}{\left(\sigma_{1}-\sigma_{3}\right)_{\mathrm{f}}}\right]^{2}
$$

où :

$E_{\mathrm{t}}$ : module tangent

$R_{\mathrm{f}}$ : facteur de rupture

Le logiciel CRISP (Briton et Gunn, 1987) est lui aussi basé sur la méthode des éléments finis, et utilise des modèles de comportement du type états critiques. Les analyses menées avec CRISP dans le cadre de ce travail, correspondent aux caractéristiques suivantes :

- le sol est modélisé par le modèle Cam-Clay modifié ; 
- les analyses drainées et non drainées sont conduites en contraintes effectives en utilisant les mêmes propriétés du sol. Dans les analyses non drainées, les surpressions interstitielles générées sont calculées à partir du module de compression volumétrique de l'eau ;

- le chargement est incrémental (module tangent);

- un élément quadrangulaire à 8 ncuds est utilisé.

Le modèle Cam-Clay modifié est basé sur les concepts de la théorie de l'état critique (Schofield et Wroth, 1968) et nécessite la détermination de cinq paramètres constitutifs pour sa mise en œuvre:

$\lambda$ : pente de la droite de consolidation vierge dans le plan e, $\log$ p':

$\mathrm{k}$ : pente de la droite de déchargement dans le plan e, $\log \mathrm{p}^{\prime}$;

$e_{c s}$ : valeur de l'indice de vides pour une valeur de p' unitaire sur la droite d'état critique dans le plan e, log p';

$M$ : pente de la droite d'état critique dans le plan (q, p');

$G$ : module de cisaillement.

La surface de charge du modèle associé est définie par:

$$
\mathrm{q}^{2}+\mathrm{M}^{2} \mathrm{p}^{2}=\mathrm{M}^{2} \mathrm{p}^{\prime} \mathrm{p}_{\mathrm{c}}
$$

avec q et $p^{\prime}$ invariants de contrainte de Cambridge et $p_{c}^{\prime}$ pression de préconsolidation isotrope.

\section{Le problème analysé}

Le travail a consisté à simuler le chargement de compression axial d'un pieu installé dans de l'argile. Le maillage utilisé est représenté sur la figure 1. Pour le pieu, on a pris en compte un béton à comportement élastique linéaire et un module d'Young égal à $25 \times 10^{6} \mathrm{kPa}$.

En ce qui concerne le sol, on a pris en compte un kaolin normalement consolidé pour lequel les paramètres correspondant au modèle de Cam-Clay modifié sont présentés dans la première colonne du tableau I.

TABLEAUI Paramètres du sol utilisés dans les analyses.

Soil parameters used in the analyses.

\begin{tabular}{l|l}
\hline Paramètres du Cam-Clay modifié & Paramètres classiques \\
\hline$\gamma^{\prime}=5,0 \mathrm{kN} / \mathrm{m}^{3}$ & $\phi^{\prime}=23^{\circ} ; \mathrm{c}^{\prime}=0,0$ \\
\hline $\mathrm{M}=0,9$ & $\mathrm{~K}_{\mathrm{o}}=1-\sin \phi^{\prime}=0,61$ \\
\hline$\lambda=0,25$ & $\alpha=0,58 ; \mathrm{A}=1,157$ \\
\hline $\mathrm{k}=0,05$ & $\beta=1,0 ; \mathrm{B}=1,0$ \\
\hline $\mathrm{e}_{\mathrm{cs}}=2,44$ & $\left(\mathrm{~S}_{\mathrm{u}} / \sigma_{\mathrm{v}}^{\prime}\right)_{\mathrm{nc}}=0,26$ \\
\hline$\Lambda=1-\lambda \mathrm{k}=0,8$ & $\mathrm{E}_{\mathrm{u}}=400 \mathrm{~S}_{\mathrm{u}} ; \mathrm{v}_{\mathrm{u}}=0,5$ \\
\hline & $\mathrm{E}^{\prime}=112,4 \sigma_{\mathrm{v}}^{\prime} ; v^{\prime}=0,2$ \\
\hline
\end{tabular}

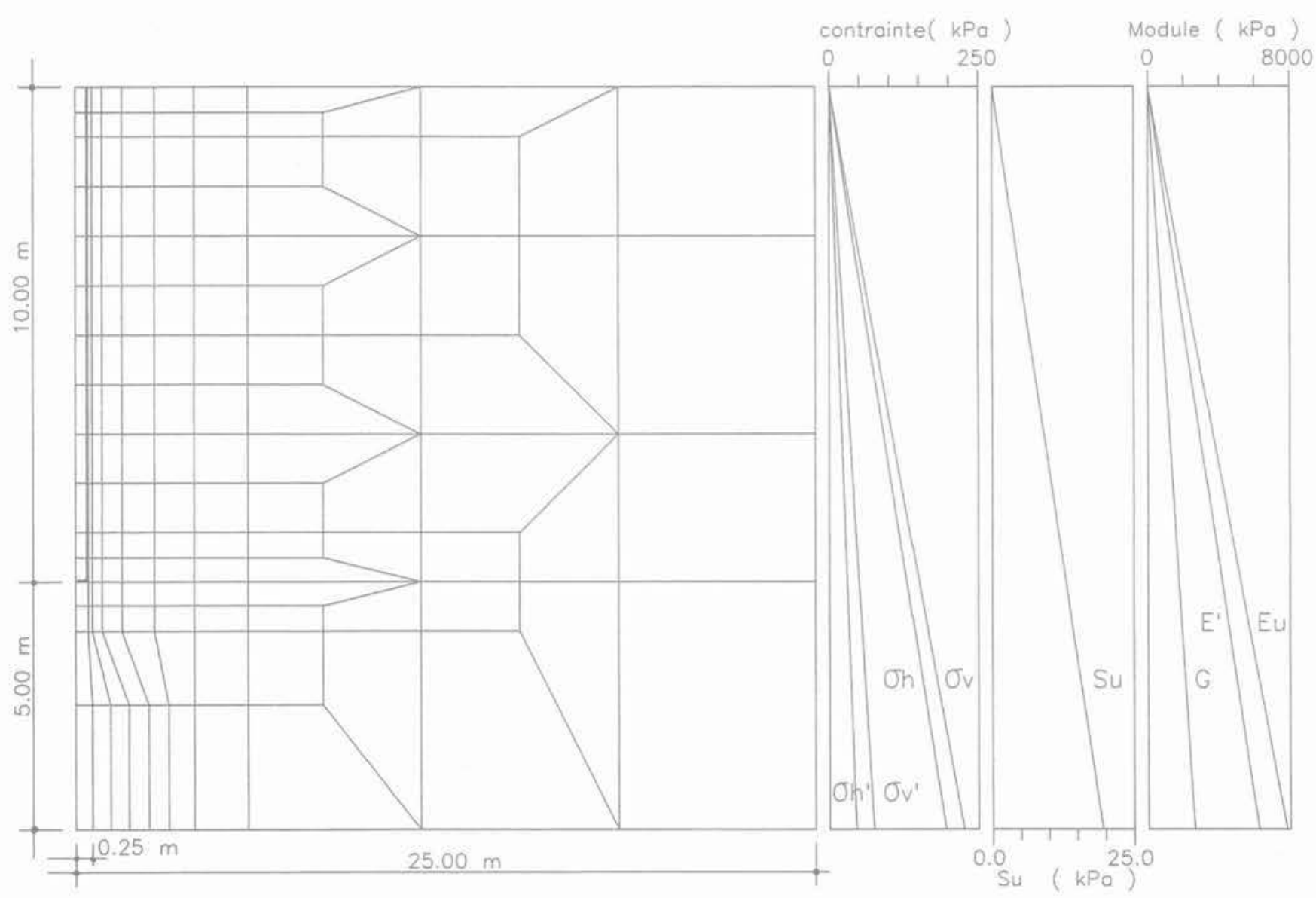

FG.1 Maillage d'éléments finis ; profils des contraintes initiales et des propriétés mécaniques. Finite element mesh, stress and property profile. 
Les paramètres classiques à prendre en compte dans le programme PROGEO ont été définis à partir de ceux adoptés pour le programme CRISP et sont présentés dans la deuxième colonne du même tableau.

Le profil des propriétés et des états de contraintes initiales avec $K_{0}=0,61$ sont présentés sur la figure 1 à partir des paramètres du tableau 1.

\section{Analyses non drainées}

Six configurations ont été étudiées, dans le cas nondrainé, qui sont présentées dans le tableau II. Malgré le fait que la valeur $K_{0}=1,0$ ne soit pas très réaliste pour une argile normalement consolidée, cè cas a été étudié pour évaluer la rotation des contraintes dans le sol au voisinage du fût du pieu.

Pour la sélection du nombre d'incréments à appliquer en tête du pieu, une étude a été faite des effets du nombre d'incréments sur la courbe charge-déplacement obtenue. Les résultats sont présentés sur la figure 2. Pour le programme CRISP, on a sélectionné une séquence de chargement avec 84 incréments inégaux (les plus petits près de la rupture), Pour le programme PROGEO on a observé que 7 incréments étaient suffisants pour produire une courbe bien stable.

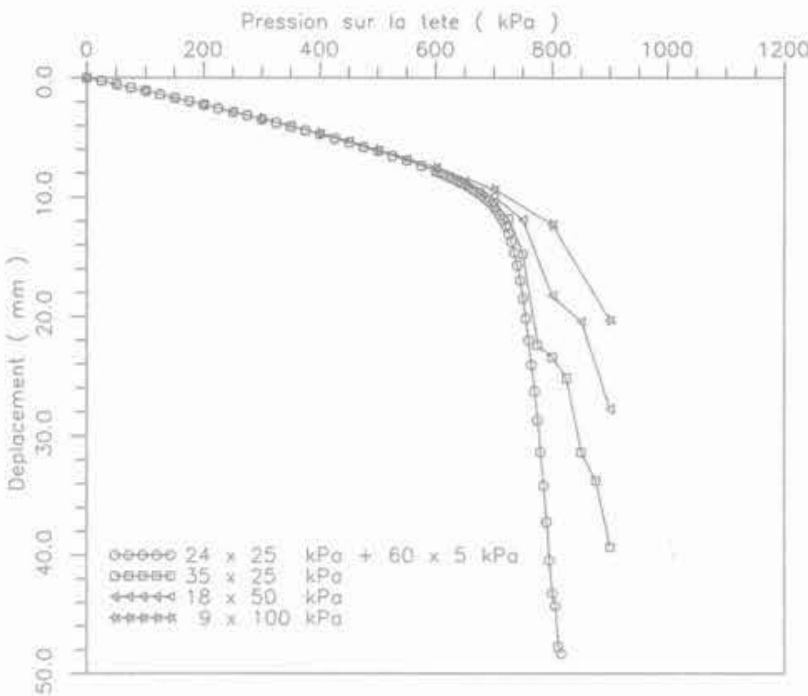

FIG. I Influence du nombre d'incréments sur la courbe charge-déplacement. CRISP Chargement non-drainé, $\mathrm{K}_{0}^{\prime}=0,61$. Influence of the number of increments on the load-settlement curve. CRISP - Undrained analyses, $\mathrm{K}_{0}^{\prime}=0.61$.

TABLEAU II Analyses non drainées. Undrained analyses.

\begin{tabular}{l|l|l} 
CAS & Programme-Analyse & $\mathrm{K}_{\circ}$ \\
U1 & PROGEO - Contraintes totales & 1,0 \\
\hline U2 & PROGEO - Contraintes totales & 0,61 \\
\hline U3 & PROGEO - Contraintes effectives & 1,0 \\
\hline U4 & PROGEO - Contraintes effectives & 0,61 \\
\hline U5 & CRISP - Contraintes effectives & 1,0 \\
\hline U6 & CRISP - Contraintes effectives & 0,61 \\
\hline
\end{tabular}

Les analyses non drainées basées sur le programme CRISP ont été initialement faites en utilisant l'élément standard (quadrilatéral de déformation linéaire). On a constaté la génération de surpressions interstitielles anormales à proximité de la pointe du pieu. Pour résoudre ce problème on a utilisé des éléments quadrangulaires pour la consolidation, qui ont donné de bons résultats.

Les courbes charge-déplacement pour $\mathrm{K}_{\mathrm{o}}=1$, 0 sont représentées sur la figure 3 . On observe que les dernières étapes du chargement, correspondant à la charge de rupture obtenue avec l'analyse en contraintes totales et avec l'analyse en contraintes effectives, sont très proches et un peu en dessous de celles obtenues avec la solution élasto-plastique.

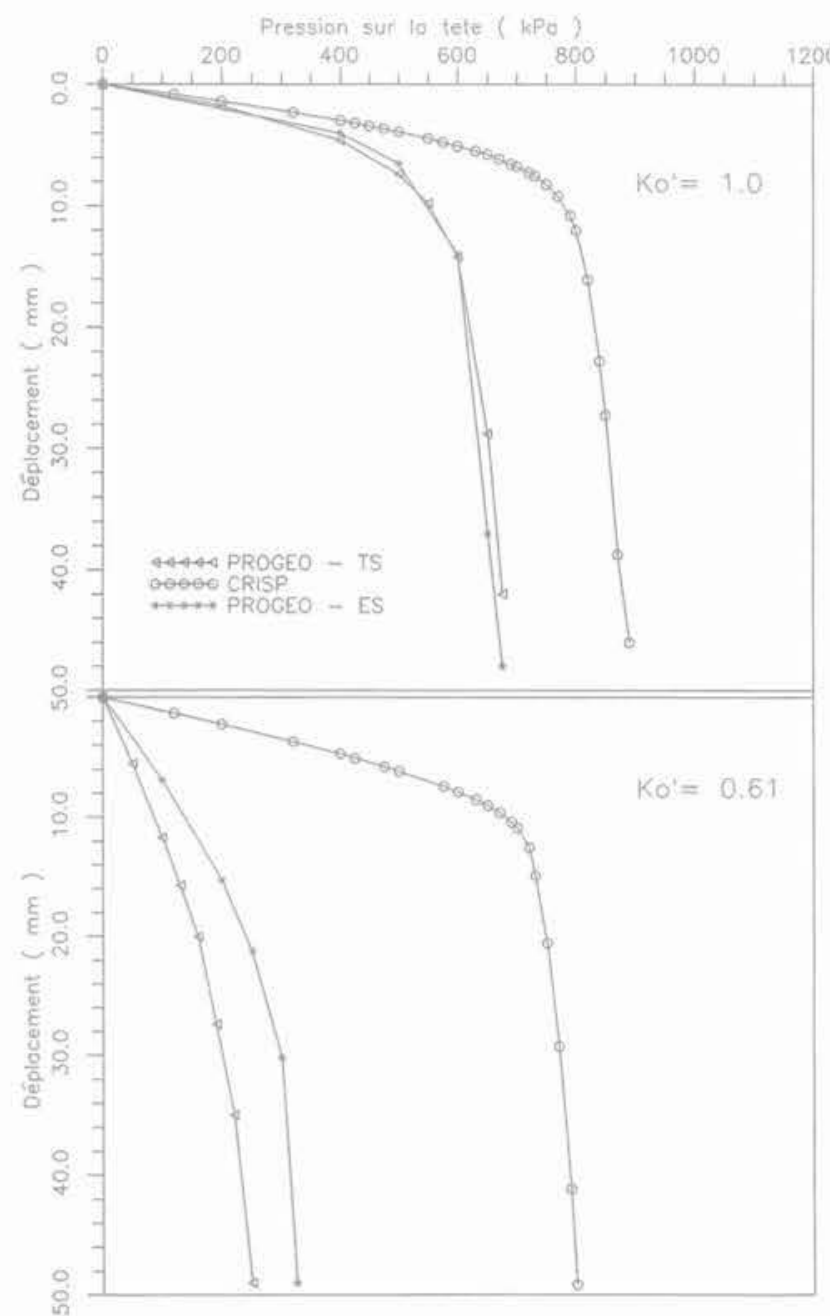

FIG. 3 Courbes charge-déplacement. Analyse non drainée. $\mathrm{K}_{\mathrm{o}}^{\prime}=1,0$ et $\mathrm{K}_{\mathrm{o}}^{\prime}=0,61$. Load-settlement curve. Undrained analyses. $\mathrm{K}_{0}^{\prime}=1.0$ and $\mathrm{K}_{0}:=0.61$.

Les chemins de contraintes suivis par les éléments au voisinage du pieu pour $\mathrm{K}_{0}=1,0$ sont montrés sur les figures 4 à 6 . En observant les chemins de contraintes totales, on peut remarquer que les conditions de chargement au voisinage du fût du pieu sont très proches d'une condition de cisaillement pur. Les chemins de contraintes effectives se déplacent vers la gauche, ceci résultant de l'évolution des surpressions interstitielles au cours du chargement. 


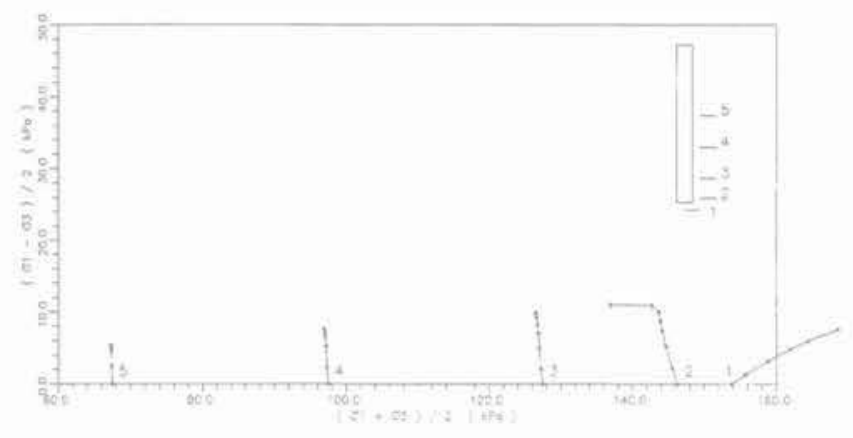

FG. 4 Chemins de contraintes totales dans des éléments au voisinage du pieu. Analyse élastique non linéaire - cas non drainé contraintes totales $-\mathrm{K}^{\prime}=1,0$.

Total stress paths in element close to the pile. Non linear elastic analysis - Undrained - Total stress $-K_{0}^{\prime}=1.0$.

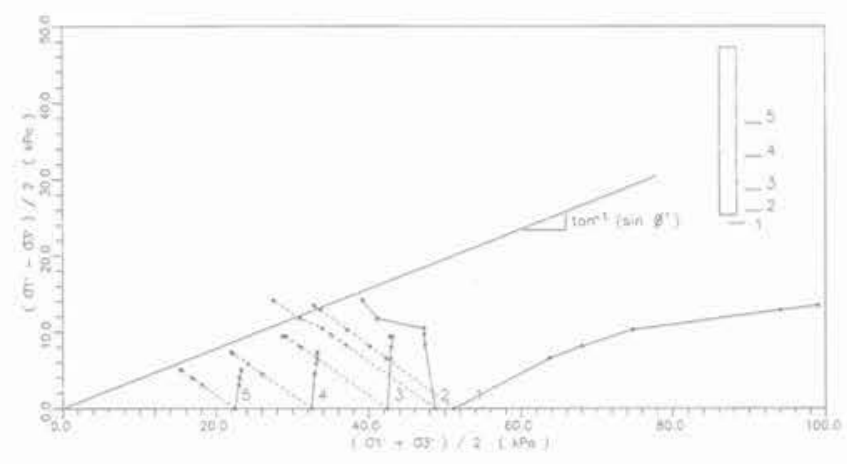

FIG. 6 Chemins de contraintes totales et effectives dans des éléments au voisinage du pieu. Analyse élastique non linéaire Cas non drainé - Contraintes effectives $\mathrm{K}_{\mathrm{o}}^{\prime}=1,0$.

Total and effective stress paths in elements close to the pile. Non linear elastic analysis Undrained - Effective stress $-\mathrm{K}_{0}^{\prime}=1.0$.

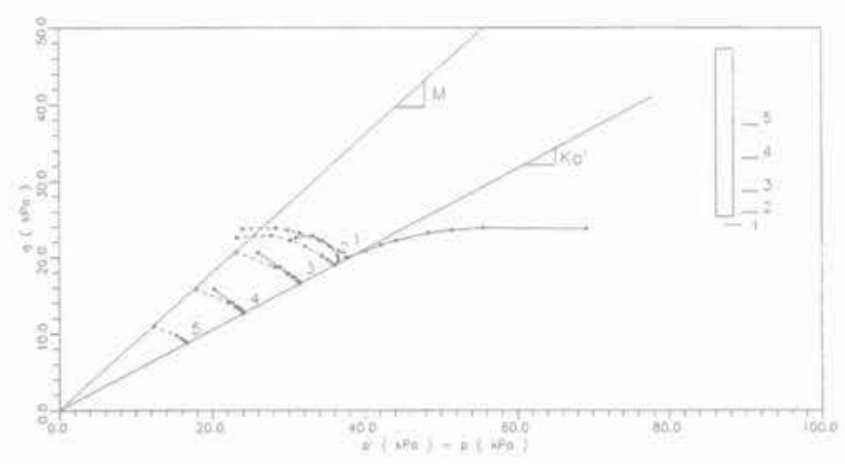

FG. 8 Chemins de contraintes totales et effectives dans des éléments ou voisinage du pieu. Analyse élasto-plastique, modèle Cam-Clay modifié - Cas non-drainé $\mathrm{K}_{\mathrm{o}}^{\prime}=0,61$.

Total and effective stress paths in elements close to the pile. Elasto-plastic analysis - Mod. Cam-Clay - Undrained $-\mathrm{K}_{\mathrm{o}}^{\prime}=0.61$.

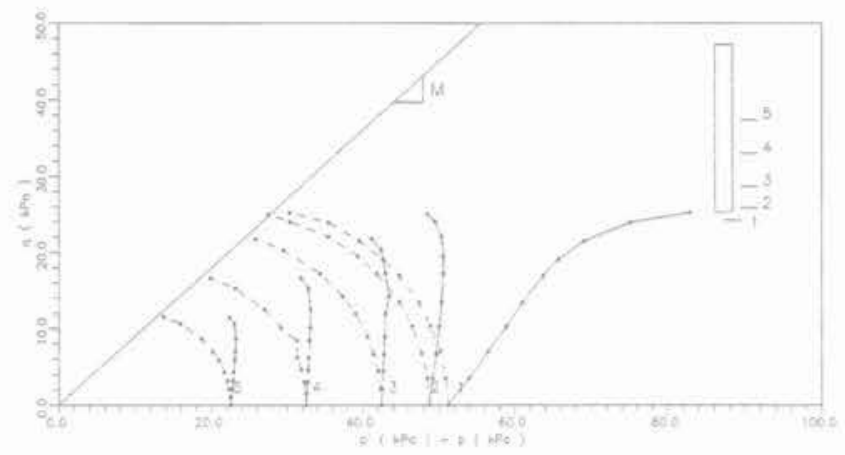

FIG, 5 Chemins de contraintes totales et effectives dans des éléments au voisinage du pieu. Analyse élasto-plastíque, modèle Cam-Clay, modifié - Cas non-drainé $\mathrm{K}_{0}^{\prime}=1,0$.

Total and effective stress paths in elements close to the pile. Elasto-plastic analysis - Mod. Cam-Clay - Undrained $-\mathrm{K}_{0}^{\prime}=1.0$.

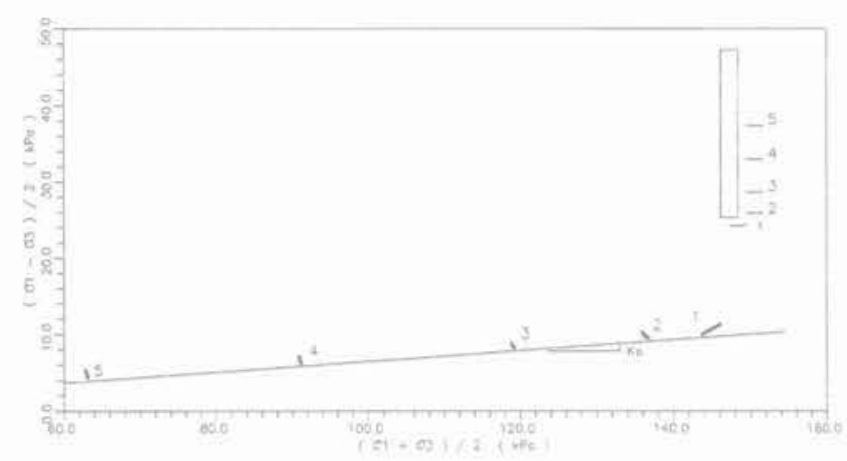

FG. 7 Chemins de contraintes totales dans des éléments au voisinage du pieu. Analyse élastique non linéaire - Cas non drainé Contraintes totales $-\mathrm{K}_{\mathrm{o}}^{\prime}=0,61$.

Total stress paths in elements close to the pile. Non linear elastic analysis - Undrained - Total stress $-\mathrm{K}_{\mathrm{o}}^{\prime}=0.61$.

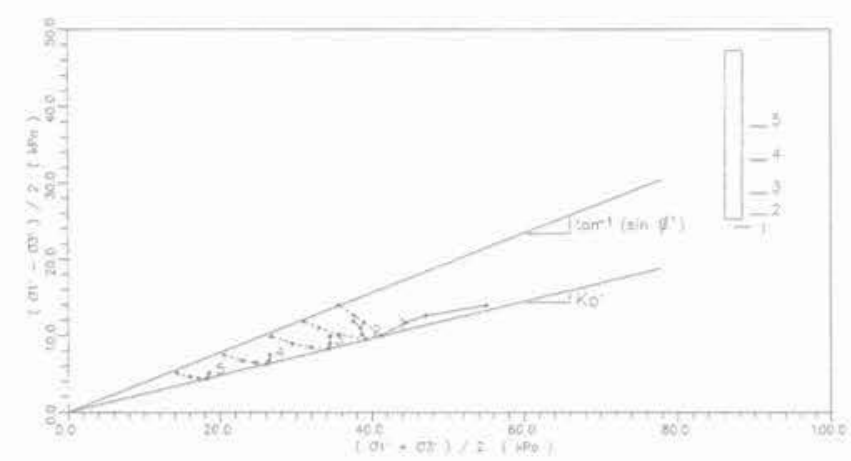

FIG. 9 Chemins de contraintes totales et effectives dans des éléments au voisinage du pieu. Analyse élastique non linéaire Cas non drainé. Contraintes effectives $\mathrm{K}_{0}^{\prime}=0,61$.

Total and effective stress paths in elements close to the pile. Non linear elastic analysis Undrained - Effective stress $-\mathrm{K}_{0}^{\circ}=0.61$. 
Les courbes de chargement obtenues pour $K_{0}=0,61$ sont représentées sur la figure $3 . \mathrm{b}$; les chemins de contraintes correspondants suivis par les éléments proches du pieu sont montrés sur les figures 7 à 9 . La charge de rupture obtenue avec la solution élastique non linéaire est beaucoup plus faible que celle obtenue avec la solution élasto-plastique. Les contraintes développées sur un élément au voisinage du fût du pieu (montré pour une profondeur de 6,5 m sur la figure 10) en explique les raisons : la contrainte de cisaillement sur le plan vertical dans une solution élastique non linéaire est beaucoup plus basse à la rupture. Dans cette solution, quand les conditions de rupture sont réalisées dans l'élément (c'est-à-dire lorsque le cercle de Mohr correspondant touche la ligne de rupture), l'élément se rompt, et la contrainte de cisaillement sur le plan vertical conserve la valeur qu'elle avait au début de la rupture. Cependant, dans une solution élasto-plastique les conditions de rupture sont réalisées avec une rotation des contraintes principales de telle sorte que la contrainte de cisaillement maximale survienne sur un plan vertical. Dans la solution élastique non linéaire, le problème est traité comme un problème à vitesse de contrainte imposée alors qu'en fait c'est un problème à vitesse de déformation imposée. Ainsi, la cinématique de la rupture n'est pas prise en compte dans le cas de l'élasticité non linéaire. D'un autre côté, avec la solution élasto-plastique, la cinématique de la rupture est prise en compte.

\section{5}

\section{Analyses drainées}

Quatre configurations ont été analysées dans le cas drainé, comme indiqué dans le tableau III.
TABLEAU III Analyses drainées. Drained analyses.

\begin{tabular}{l|l|l} 
CAS & Programme & $\mathrm{K}_{0}$ \\
D1 & PROGEO & 1,0 \\
\hline D2 & PROGEO & 0,61 \\
\hline D3 & CRISP & 0,1 \\
\hline D4 & CRISP & 0,61 \\
\hline
\end{tabular}

Les courbes charge-déplacement obtenues sont présentées sur la figure 11 pour $K_{0}=1,0$ et $K_{0}=0,61$. Pour $\mathrm{K}_{0}=1,0$ les charges de rupture obtenues avec les deux logiciels sont très proches alors que pour $K_{0}=0,61$ la charge de rupture obtenue avec la solution élastique non linéaire est bien plus faible que celle correspondant au modèle de Cam-Clay modifié.

Les chemins de contraintes suivis pour des éléments situés au voisinage du pieu sont montrés sur les figures 12 à 15 . Les conditions de chargement avec une solution élastique non linéaire sont de type cisaillement pur (contrainte moyenne constante alors que dans la solution élasto-plastique on observe une réduction de la contrainte moyenne d'environ $20 \%$.

Comme dans le cas non drainé, le frottement latéral obtenu pour $K_{0}=0,61$ pour la solution élastique non linéaire est considérablement plus faible que celui observé pour la solution élasto-plastique, et ceci est dû à nouveau à la rotation des contraintes principales comme conséquences de la cinématique de rupture (Fig. 16).
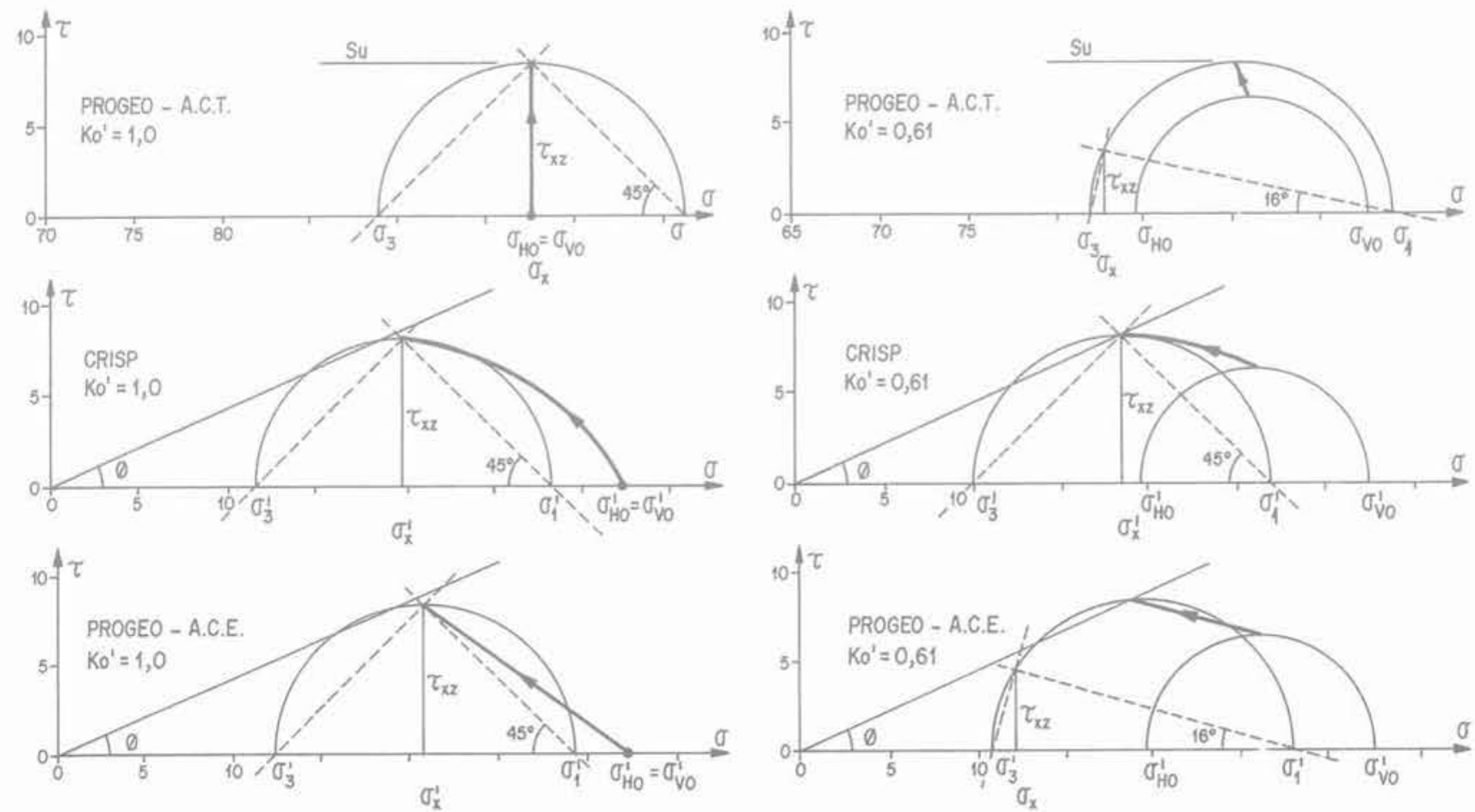

F1G. 10 Développement des contraintes dans un élément au voisinage du fút pendant un chargement non-drainé. Stress development in an element adjacent to the pile shaft (depth $=6.50 \mathrm{~m}$ ) in undrained loading. 


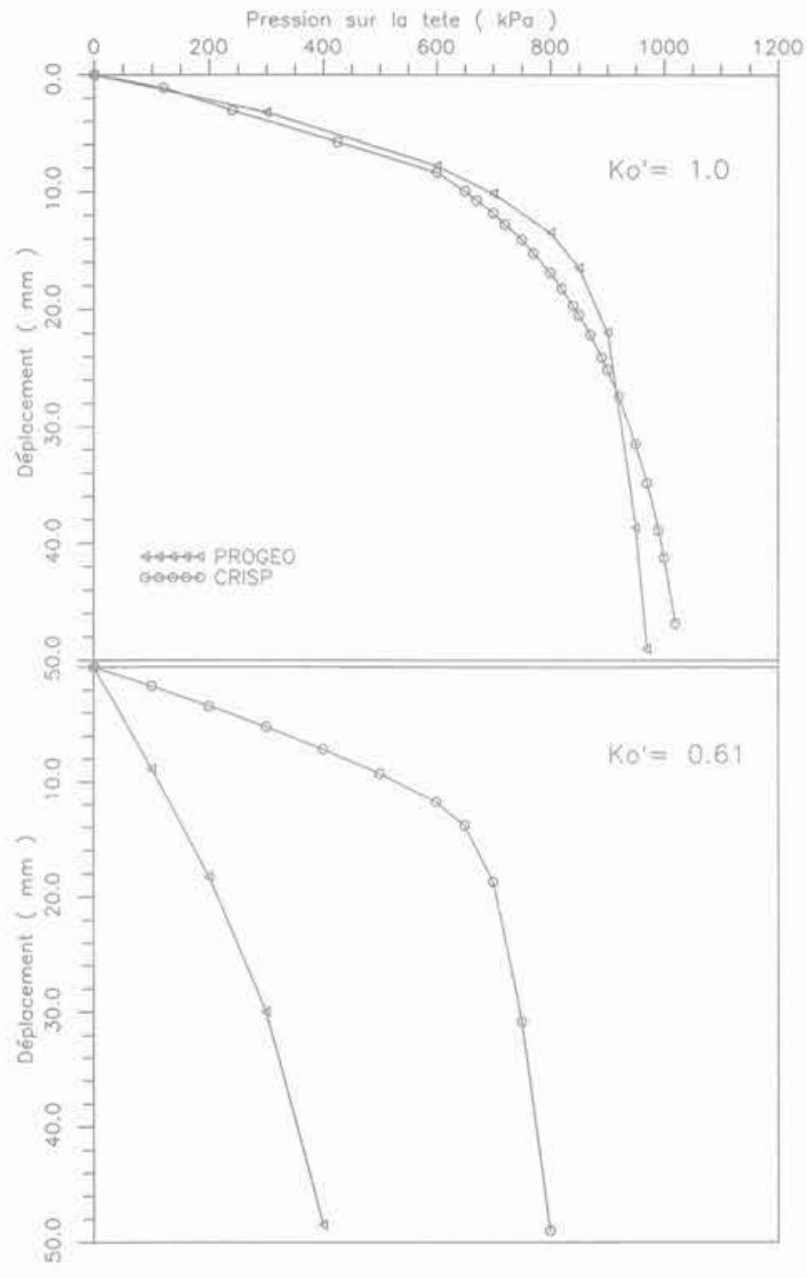

FIG. 11 Courbes charge - déplacement. Analyse drainée. $K_{0}^{\circ}=1,0$ et $K_{0}^{\prime}=0,61$.

Load-settlement curve, Drained analyses. $\mathrm{K}_{0}^{\prime}=$ 0.1 and $K_{0}=0.61$.

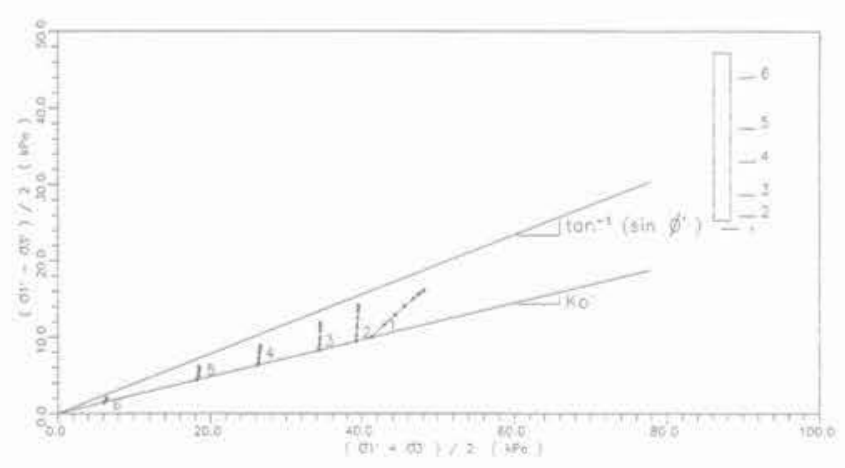

FIG. 14 Chemins de contraintes effectives dans des éléments au voisinage du pieu.

Analyse élastique non-linéaire, cas drainé $-\mathrm{K}_{0}^{\prime}=0,61$.

Effective stress paths in elements close to the pile. Non linear elastic analysis - Drained $\mathrm{K}_{0}^{\prime}=0.61$.

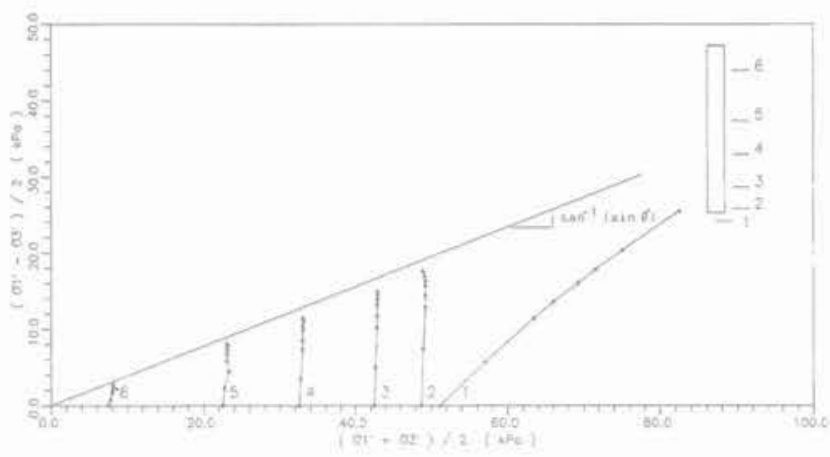

FIG. 12 Chemins de contraintes effectives dans des éléments au voisinage du pieu. Analyse élastique non linéaire $-K_{0}^{\prime}=1,0$, Effective stress paths in elements close to the pile. Non linear elastic analysis - Drained $\mathrm{K}_{0}=1.0$.

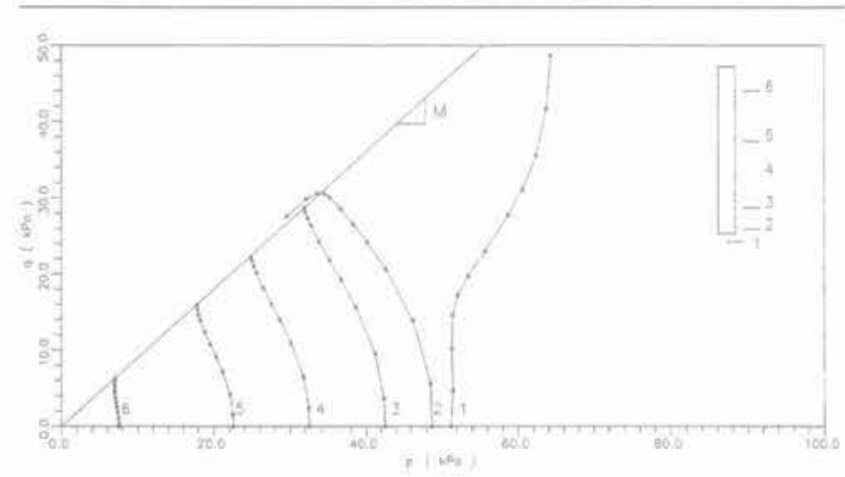

FiG. 13 Chemins de contraintes effectives dans des éléments au voisinage du pieu. Analyse élasto-plastique, modèle CamClay, modifié. Cas drainé $-\mathrm{K}_{\mathrm{o}}^{\prime}=1,0$. Effective stress paths in elements close to the pile. Elasto-plastic analysis-Mod, Cam-ClayDrained $-\mathrm{K}_{0}^{\prime}=1.0$.

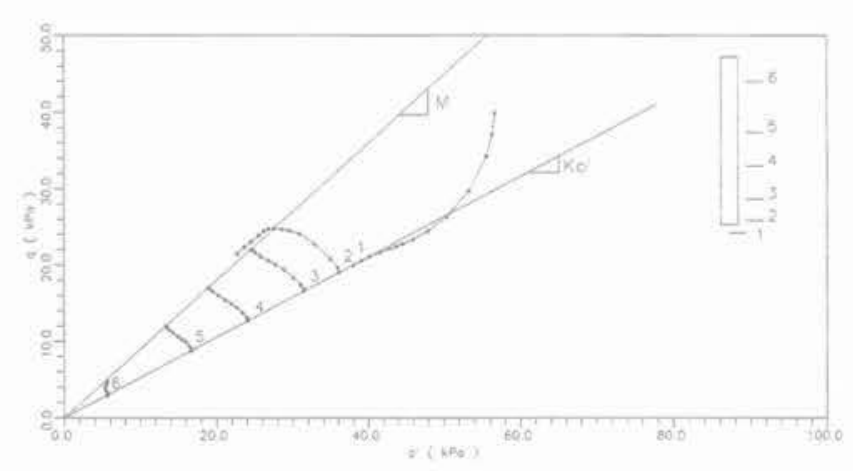

FG. 15 Chemins de contraintes effectives dans des éléments au voisinage du pieu. Analyse élasto-plastique, modèle CamClay, modifié - Cas drainé $-\mathrm{K}_{0}^{\prime}=0,61$. Effective stress paths in elements close to the pile. Elasto-plastic analysis - Mod. Cam-Clay Drained $-\mathrm{K}_{0}^{\prime}=0.61$. 

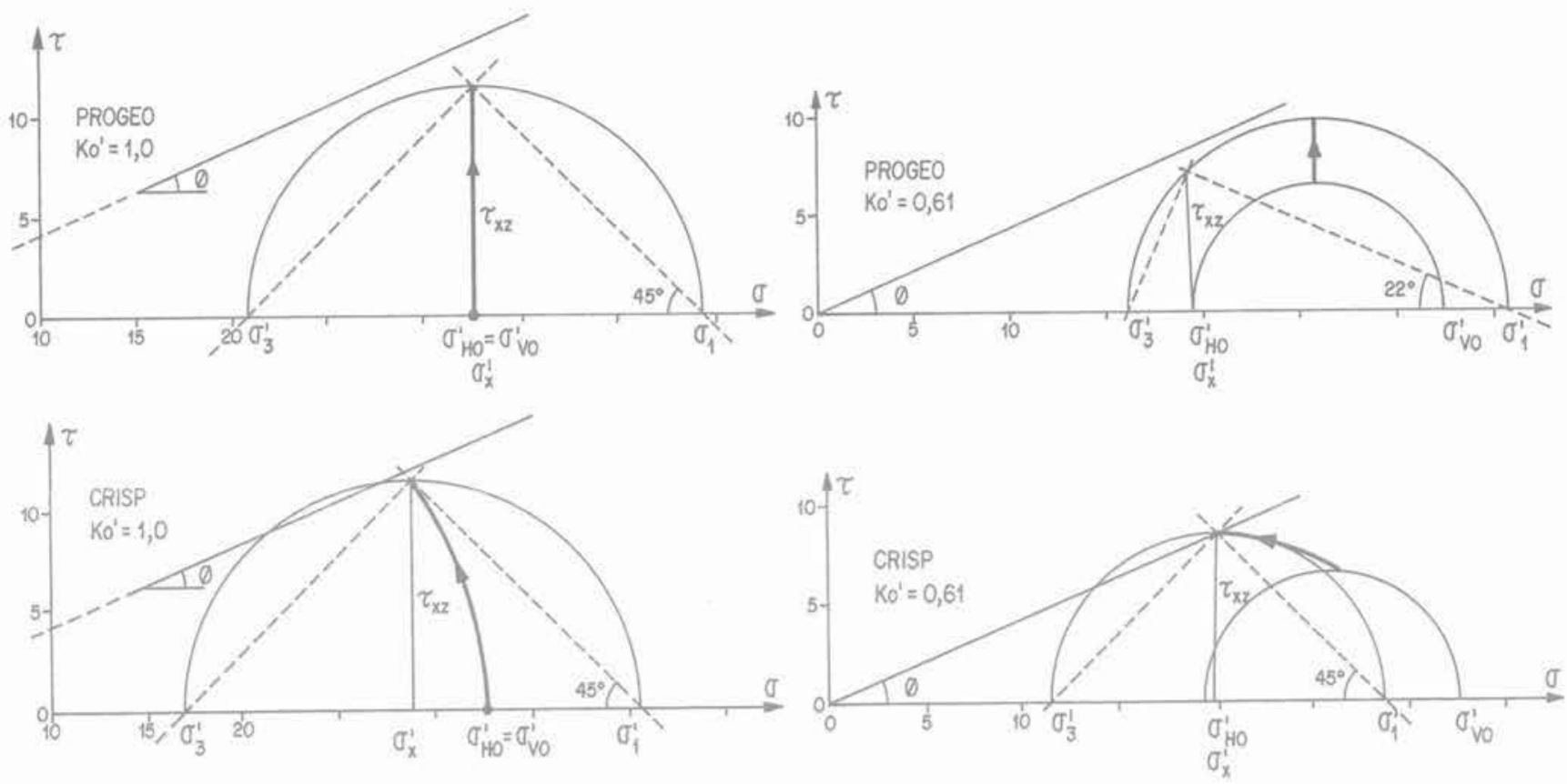

FIG. 16 Développement des contraintes dans un élément au voisinage du fût pendant un chargement drainé. Stress development in an element adjacent to the pile shaft (depth=6.50m) in drained loading.

\section{6}

\section{Influence de la surconsolidation dans le cas du chargement non drainé}

En vue de mettre en évidence l'effet de la surconsolidation sur le frottement latéral calculé, trois cas nondrainés représentant des argiles surconsolidées ont été analysés, en plus du cas U1. Les différents cas étudiés sont présentés dans le tableau IV. On a considéré à nouveau le pieu installé d'une façon idéale. L'état initial de contraintes est basé sur une valeur de $K_{0}$ calculée comme l'a proposé Parry (1970) :

$$
\mathrm{K}_{0}=(1-\sin \phi) \text { OCR } \phi \mathrm{rad}
$$

TABt.EAUIV Analyses non-drainées avec différents OCR.

Undrained analyses with different OCRs

\begin{tabular}{l|l|l|l} 
CAS & Programme-Analyse & OCR & $\mathrm{K}_{\circ}$ \\
$\mathrm{U} 1$ & CRISP - Non-drainée & 1,0 & 0,61 \\
\hline $\mathrm{O} 2$ & CRISP - Non-drainée & 1,5 & 0,72 \\
\hline $\mathrm{O} 2$ & CRISP - Non-drainée & 2,0 & 0,81 \\
\hline $\mathrm{O} 3$ & CRISP - Non-drainée & 3,0 & 0,95 \\
\hline
\end{tabular}

Sur la figure 17, les chemins de contraintes effectives sont représentés pour un élément au voisinage du pieu à une profondeur de $6,50 \mathrm{~m}$ (il s'agit de l'élément 4 qui apparait sur les diverses figures représentant les chemins de contrainte). Pour les sols ayant un degré de surconsolidation supérieur à 1,0 les chemins de contraintes effectives sont verticaux quand le chemin de contrainte est à l'intérieur de la surface d'écoulement ; quand la surface d'écoulement est atteinte, le chemin de contrainte change de direction et se dirige vers l'état critique. Ceci est en accord avec le modèle Cam-Clay modi- par le modèle que les conditions cinématiques de la rupture. Dans toutes les analyses réalisées la contrainte de cisaillement maximale s'est développée sur un plan vertical et était approximativement égale à la résistance au cisaillement non drainée du sol.

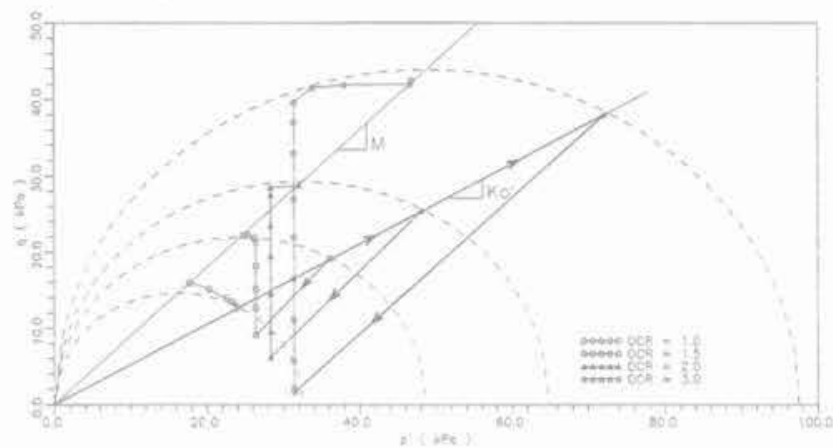

FIG. 17 Chemins de contraintes effectives dans un élément au voisinage du pieu pour différents facteurs de surconsolidation (Modèle Cam-Clay). Profondeur $=6,50 \mathrm{~m}$. $\mathrm{K}_{\mathrm{o}}^{\prime}=(1-\sin \phi) \mathrm{OCR}^{\phi(\mathrm{rad})}$

Effective stress paths in an element close to the pile staft for different OCRs - Elasto-plastic analysis (Mod. Cam-Clay) Depth $=6.50 \mathrm{~m}$, $K_{0}^{\prime}=(1-\sin \phi) O C R^{\phi}$ (rad) 
tallation du pieu, le sol près du pieu est totalement remanié et, qu'après la dissipation des surpressions interstitielles engendrées, on observe une augmentation de la résistance au cisaillement non drainée $\left(S_{14}\right)$ par rapport à la résistance non drainée initiale $\left(\mathrm{S}_{\mathrm{uo}}\right)$. L'état de contrainte final est donné par $\sigma_{\mathrm{r}}^{\prime}=5 \mathrm{~S}_{\mathrm{uo}}$ et $\sigma_{\theta}^{\prime}=3 \mathrm{~S}_{\mathrm{uo}}$ indépendamment de la valeur de l'OCR avant l'installation du pieu.

L'hypothèse de l'état de déformations planes conduit à des valeurs des contraintes verticales beaucoup plus grandes que les contraintes géostatiques pour les sols surconsolidés. Au niveau du terrain et à long terme, on s'attend à un relâchement de $\sigma_{y}^{\prime}$ accompagné ou non d'un relâchement de $\sigma_{r}^{\prime}$ et de $\sigma_{\theta}^{\prime}$. Le chemin de contraintes pour deux sols présentant des OCR différents près du pieu à la même profondeur, dans les phases de repos, d'installation, de consolidation et à long terme sont montrés sur la figure 18. Dans cette situation, les sols ont des valeurs de $S_{u}$ différentes. Dans les deux cas de chemins de contraintes, les points A, B, $C$ (ou $A^{\prime}, B^{\prime}, C$ ) représentent les phases de repos, d'installation et de consolidation, suggérées par Randolph et al. (1979). Les points D, D' et E' représentent des états possibles de contraintes à long terme en admettant que la contrainte verticale effective $\sigma_{\mathrm{y}}^{\prime}$ devienne égale aux contraintes géostatiques.

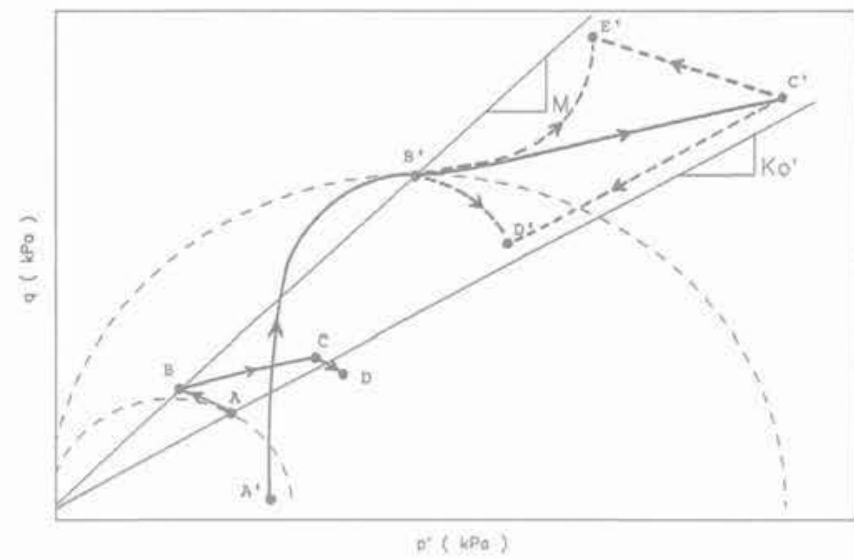

FG. 18 Chemins de contraintes effectives dans un élément au voisinage du pieu par Randolph et al. (1979) (points A, B, C; A: $\left.B^{\prime}, C^{\prime}\right)$ et avec la considération de la relaxation de la contrainte verticale (points D; D', E').

Effective stress paths in an element adjacent to the pile staft as predicted by Randolph and al (1979) (points A, B, C; A', B', C') and

considering relaxation of vertical stress (points D; D', E'),

Pour un sol normalement consolidé, I'état de contrainte proposé par Randolph et al. (1979) (point C) est proche de l'état de contrainte à long terme (point D). Pour un sol surconsolidé, l'état de contrainte proposé (point C') amène à un état de contrainte beaucoup plus grand que l'état de contrainte géostatique. Dans cette situation, le sol est soumis à un relâchement de $\sigma^{\prime}$, avec un relâchement proportionnel de $\sigma_{r}^{\prime}$ et de $\sigma_{\theta}^{\prime}$, se dirigeant vers le point $D^{\prime}$, ou, alternativement, sans relâchement de $\sigma_{r}^{\prime}$ et de $\sigma_{\theta}^{\prime}$ se dirigeant vers le point $E^{\prime}$. Ce relâchement peut être rapide par rapport à la consolidation (chemins B' 'et B'E) ou peut être lent par rapport à la consolidation (chemin $\mathrm{B}^{\prime} \mathrm{C}^{\prime} \mathrm{D}^{\prime}$ et $\mathrm{B}^{\prime} \mathrm{C}^{\prime} \mathrm{E}$ ). Il existe deux possibilités qui dépendent de la relation entre les vitesses de consolidation et de relâchement des contraintes : le sol subit une augmentation de résistance au cisaillement non drainée $\mathrm{S}_{u}$ et termine dans le domaine surconsolidé, ou n'a pas d'augmentation de $S_{u}$ et termine dans le domaine normalement consolidé.

Les chemins de contraintes présentés sur la figure 18 sont des cas extrêmes des possibilités étudiées. En admettant n'importe quel type de relation entre les vitesses de consolidation et de relâchement des contraintes, un sol surconsolidé peut, à long terme, se trouver en I, point d'une ligne liant D' à E'. La théorie de l'expansion d'une cavité cylindrique avec l'hypothèse d'un état de déformations planes sur l'axe ne satisfait pas les conditions de long terme pour les sols surconsolidés.

\section{8}

\section{Conclusion}

La comparaison de deux solutions numériques basées sur l'utilisation des éléments finis, et de deux modèles de comportement, l'un élastique non linéaire et l'autre élasto-plastique, a permis de mettre en évidence des différences considérables entre les analyses drainées et non drainées de pieux chargés axialement et installés dans une argile surconsolidée.

Pour le modèle élastique non linéaire, le frottement latéral maximal mobilisable le long du pieu est fortement dépendant de l'état de contraintes initial (c'est-à-dire de la valeur de $\mathrm{K}_{\mathrm{o}}$ ), alors que pour le modèle élastoplastique (Cam-Clay modifié) la contrainte de cisaillement maximale est approximativement indépendante de la valeur de $\mathrm{K}_{0}$. Pour ce dernier type de solution, la rotation des contraintes principales est toujours proche de $45^{\circ}$, la contrainte de cisaillement maximale survenant sur le plan vertical et étant approximativement égale à la contrainte de cisaillement maximale. Pour la solution élastique non linéaire, la rotation des contraintes principales est inférieure à $45^{\circ}$ pour une valeur de $K_{0}$ différente de l'unité, et la contrainte de cisaillement sur le plan vertical est plus faible que la contrainte de cisaillement maximale. Cette différence peut être expliquée par une meilleure adéquation du modèle élasto-plastique au problème étudié. Ce dernier modèle satisfait en effet les conditions cinématiques du problème qui n'est pas un problème à vitesse de contrainte imposée mais à vitesse de déformation imposée, le pieu étant beaucoup plus rigide que le sol et présentant seulement des déformations verticales.

En se basant sur les résultats de cette étude, on peut observer que la valeur de $\alpha$ dans la « Méthode Alpha ») n'est pas affectée par l'état de contraintes avant le chargement du pieu. La valeur de $\alpha$ est affectée seulement par des variations de résistance au cisaillement du sol pendant l'installation et la consolidation du sol qui s'en suit.

Le programme CRISP, basé sur l'utilisation du modèle Cam-Clay modifié, donne de bons résultats pour l'argile surconsolidée avec des valeurs de OCR comprises entre 1,0 et 3,0 .

Les variations de contrainte induites dans le sol par l'installation du pieu et la dissipation des surpressions interstitielles résultantes peuvent être représentées par un modèle d'expansion de cavité cylindrique avec une hypothèse de déformations planes par rapport à l'axe du pieu. Cependant, l'état de contraintes à long terme prévu pour des sols surconsolidés est incompatible avec le relâchement des contraintes verticales dû à la surface libre. 
Almeida M.S.S., Britto A.M.. Parry R.H.G. - Numerical Modelling of a Centrifuged Embankment on Solft Clay, Canadian Geotechnical Journal, vol. 23, 1986.

Britto A.M., Gunn M.J. - Critical State Soil Mechanics Via Finite Elements, Ellis Horwood Limited, 1987.

Brugger P.J, - Numerical Analysis of Piles Subjected to Axial Com. pression (en portugais), M. Sc. thesis, COPPE-UFRJ, Rio de Janeiro, 1991

Burland J.B. - Shaft Friction of Piles in Clay, Ground Engineering, vol. 3, 1973
Chandier R.J. - The Shaft Friction of Piles in Cohesive Soils in Terms of Effective Stress, Civil Engineering and Public Works Review, vol. 63, 1968

Lopes F.R. - The Undrained Bearing Capacity of Piles and Plates Studied by the Finite Element Method, PhD. Thesis, Imperial College of Science and Technology, University of London. 1979

Martins J.P. Potts D.M. - A Numerical Study of Skin Friction Around Driven Piles, Proceedings, Numerial Methods in Offshore Pilling, 1983.
McClelland B., Focht J.A., Emrich W.J. - Problems in Design and Installation of Offshore Piles, Journal of the Soil Mech. and Found. Div, ASCE, 1969.

Parry R.H.G., Swain C.W. - A Study of Skin Friction on Piles in Stiff Clay, Ground Engineering, vol. 10, 1977-b.

Potts D.M. Martins J.P. - The Shear Resistance of Axially Loaded Piles in Clay - A Theoretical Study, Geotechnique, 1984.

Randolph M.F., Carter J.P., Wroth C.P. - Driven Piles in Clay - The
Effects of Installation and Subsequent Consolidation, Geotechnique, $\mathrm{N}^{\circ} 4,1979$.

Schofield A.N., Wroth C.P. - Critical State Soll Mechnics, London, McGraw Hill Editors, 1968

Tomlinson M.J. - The Adhesion of Piles Driven in Clay Soils, Procedings, 4th Int. Conf. on Soil Mech. and Found. Eng., Lon. don, 1957.

Wroth C.P. - Discussion, Proceedings Specialty Conference on Performance of Earth and Earth-supported Structures, vol. 3, ASCE, New York, 1972. 